\title{
Hepatitis C: una aproximación clínica y epidemiológica en Cali, Colombia
}

\section{Clinical and epidemiological approach to Hepatitis C in Cali, Colombia}

Carlos A. Rojas, MD, ${ }^{*}$ Angélica Tobón, MD, ${ }^{2}$ Mauricio Sepúlveda, MD, ${ }^{3}$ Nelson Rojas, MD, ${ }^{4}$ Diego Jiménez, MD. ${ }^{5}$

\footnotetext{
1 Departamento de Medicina Interna,

Departamento de Medicina Interna,
Gastroenterología, Fundación Clínica Valle del Lili. Cali, Colombia.

2 Especialización en medicina interna, Facultad de Ciencias de la Salud, Universidad ICESI. Cali, Colombia.

3 Departamento de Medicina Interna, Gastroenterología, Fundación Clínica Valle del Lili. Cali, Colombia.

4 Programa de Medicina, Facultad de Ciencias de la Salud, Universidad ICESI. Cali, Colombia.

5 Departamento de Medicina Interna,

Gastroenterología y Hepatología, Fundación Clínica Valle del Lili. Cali, Colombia.

*Correspondencia: crojo16@yahoo.com.
}

\begin{abstract}
Resumen
Introducción: en Colombia, la infección por el virus de la hepatitis C (VHC) representa alta morbilidad y elevados costos. Con la llegada de nuevos tratamientos más efectivos, se hace necesario conocer las características propias de esta población para su adecuado uso. Objetivos: describir las características epidemiológicas y clínicas de los pacientes con VHC manejados en un centro de referencia en enfermedades hepáticas. Materiales y métodos: se realizó un estudio descriptivo de corte transversal en una población de adultos con diagnóstico serológico de VHC entre el 2011 y el 2016. Resultados: se evaluaron 214 historias clínicas de pacientes con diagnóstico serológico confirmado de VHC. La mediana de edad fue de 59 años y el $62 \%$ fue de sexo femenino. El genotipo se reportó en 114 pacientes, el $75 \%$ presentó genotipo $1 \mathrm{~B}$. El 36,9 $\%$ de los pacientes había recibido algún hemoderivado y el $5 \%$ tenía tatuajes. La prevalencia de cirrosis fue del $29,4 \%$ y de hepatocarcinoma fue del 3,3\%. El 1,8 \% y el 5,1\% de los pacientes presentó coinfección con el virus de la hepatitis $B(\mathrm{VHB})$ y virus de la inmunodeficiencia humana $(\mathrm{VIH})$, respectivamente. Conclusión: los factores determinantes de la infección por el VHC en Cali presentan un comportamiento clínico similar al que reporta la literatura científica a nivel mundial, lo que obliga a enfatizar en la prevención de la población en riesgo. El genotipo 1B continúa siendo el más frecuente en nuestro medio, lo que hace a esta población susceptible a los nuevos tratamientos.
\end{abstract}

\section{Palabras clave}

Virus de la hepatitis C, hepatocarcinoma, cirrosis, genotipo, virus de la hepatitis B, trasfusiones, agentes antivirales directos.

\begin{abstract}
Introduction: In Colombia, Hepatitis C virus infections have high rates of morbidity and high costs. The advent of new more effective treatments has produced a need for better knowledge of this population's characteristics to allow their proper use. Objectives: The objective of this study is to describe the epidemiological and clinical characteristics of patients with hepatitis $C$ at a referral center for liver diseases. Materials and methods: We conducted a cross-sectional descriptive study of a population of adults with serological diagnoses of hepatitis $\mathrm{C}$ between 2011 and 2016. Results: We evaluated 214 clinical records of patients with confirmed serological diagnoses of hepatitis C. Their median age was 59 years, and $62 \%$ were women. The HCV genotypes of 114 patients were reported: $75 \%$ had genotype $1 \mathrm{~B}$. Transfusions of one or another type of blood product had been administered to $36.9 \%$ of the patients, and $5 \%$ had tattoos. The prevalence of cirrhosis was $29.4 \%$ while that of hepatocellular carcinoma was $3.3 \%$. Hepatitis B virus coinfections were found in $1.8 \%$ of these patients, and $5.1 \%$ of the patients had human immunodeficiency virus coinfections. Conclusion: The determinants of hepatitis $C$ virus infection in Cali are similar to those reported in scientific literature worldwide and requires emphasis on prevention in the at-risk population. Genotype $1 \mathrm{~b}$ continues to be the most frequent in our environment which makes this population susceptible to new treatments.
\end{abstract}

\section{Keywords}

Hepatitis C virus, hepatocellular carcinoma, cirrhosis, genotype, hepatitis B virus, transfusions, direct antiviral agents. 


\section{INTRODUCCIÓN}

El virus de la hepatitis C (VHC) pertenece a la familia Flaviviridae y genera infecciones agudas que frecuentemente se vuelven crónicas en el $50 \%$ al $85 \%$ de los casos. Se conocen 6 genotipos y, al menos, 50 subtipos diferentes. La principal vía de transmisión de este virus es la parenteral ( $85 \%)$. Existen otras vías menos frecuentes como la sexual (menos del $2 \%$ ) y la vertical (10 \%) (1). Entre el $5 \%$ y el $30 \%$ de los individuos con infección crónica desarrolla cirrosis. En países desarrollados como Estados Unidos, la infección por VHC es la causa más importante de enfermedad hepática, así como la indicación más frecuente de trasplante hepático (2).

El tiempo que tarda un individuo en desarrollar cirrosis posterior a la infección fue estimado en una revisión sistemática de Hla-Hla Thein y colaboradores, en la que se evaluaron 111 estudios y se encontró una prevalencia de cirrosis 20 años después de la infección en cerca del $16 \%$ de los estudios analizados (3).

Se considera que un tercio de los pacientes con carcinoma hepatocelular presentó infección por el VHC. Una vez se diagnostica la cirrosis, se estima que el riesgo de presentar carcinoma hepatocelular está entre el $0 \%$ y el $3 \%$ por año en varios reportes $(4,5)$.

La coinfección con el VHC y el virus la hepatitis B (VHB) no es infrecuente, dado que comparten modos de transmisión similares. La ocurrencia de estas infecciones simultáneas es mucho más prevalente en áreas en las que ambos virus son endémicos y entre grupos de personas con alto riesgo de contagio parenteral. La importancia clínica de diagnosticar la coinfección VHC/VHB radica tanto en el pronóstico, dado que estos pacientes presentan más desenlaces adversos, como en las opciones de tratamiento (6).

El virus de la inmunodeficiencia humana (VIH) genera otra de las infecciones más comúnmente encontradas en pacientes con el VHC. Aproximadamente, el $10 \%$ de los pacientes VHC positivos también es positivo para el VIH, y de los pacientes VIH (+), aproximadamente el $25 \%$ es positivo para el VHC. Esta asociación se ha observado principalmente en grupos de riesgo, como los usuarios de drogas endovenosas. Se sabe que la presencia de ambos virus facilita la transmisión de VHC por vía sexual. De igual modo, condiciona un curso más agresivo de la infección, con enfermedad hepática en estadio final como la principal causa de muerte en esta población, por lo que se hace fundamental el tamizaje oportuno y adecuado de estos pacientes (7).

Globalmente se estima que, en el 2005, más de 184 millones de personas tuvieron anticuerpos positivos para el VHC. Con prevalencias moderadas entre $1,5 \%$ y el 3,5 $\%$ en América Latina (8). Según el Centers for Disease Control and Prevention (CDC), la incidencia total esti- mada en el 2010 fue de 0,3 por cada 100000 habitantes en Estados Unidos (9, 10).

Los estudios epidemiológicos en Colombia presentan datos generales. Las estadísticas de SIVIGILA señalan datos específicos de subgrupos en riesgo, evidenciando mayor incidencia de la infección entre los receptores de transfusiones sanguíneas (18,9\%), los homosexuales (12,8 $\%)$, las personas con múltiples parejas sexuales $(12,6 \%)$ y los usuarios de drogas endovenosas (9,1\%) (11). Pero no incluyen información sobre los genotipos y los desenlaces más frecuentes de la enfermedad (cirrosis, hepatocarcinoma y trasplantes).

La incidencia de la infección fue impactada por la implementación del tamizaje obligatorio para VHC para 1993 en los bancos de sangre. Previo a esta medida, existía una probabilidad entre el $0,74 \%$ y el $0,67 \%$ de recibir una unidad de sangre infectada o una infección transmitida por una transfusión, respectivamente. Estas cifras situaron a Colombia como uno de los países con mayor riesgo de transmisión de la infección en América Latina. En 2002 se evidenció un aumento al 99,7 \% de la tamización para VHC en los bancos de sangre, con una reducción notable del riesgo $(0,0024 \%)$ de recibir sangre infectada (12).

Sobre Cali y el Valle del Cauca hay información escasa sobre el comportamiento epidemiológico y clínico de la enfermedad, por lo que este estudio permite identificar las complicaciones más frecuentes, factores asociados con el desarrollo de esta entidad y genotipos prevalentes.

\section{MATERIALES Y MÉTODOS}

\section{Tipo de estudio}

Se realizó un estudio descriptivo transversal. Los criterios de inclusión fueron pacientes adultos de ambos sexos que tuvieran un diagnóstico de VHC dado por la presencia de anticuerpos totales contra $\mathrm{VHC}$ y o carga viral positiva, y que consultaron al servicio de gastrohepatología en la institución.

Se realizó la revisión de las historias clínicas de los pacientes en el sistema informático de la institución $\left(\mathrm{SAP}^{1}\right)$ con diagnósticos por códigos del CIE-10 de VHC y hepatitis virales, entre 2011 y 2016 se registró la información en el formato de recolección de datos diseñado para tal fin.

\section{Análisis de datos}

La información clínica, los resultados de exámenes y los datos epidemiológicos se consignaron en una base de datos electrónica. Los datos se resumieron usando como promedios la desviación estándar o la mediana, y los rangos intercuartílicos según correspondiera. En el análisis univariado se evaluó la distribución de las variables numé- 
ricas mediante la prueba de Shapiro-Wilk. Las variables cualitativas se expresaron como proporciones. Los datos se analizaron con el paquete estadístico $\operatorname{Stata}^{\mathrm{O}}$ (Stata Corp, 2011, Stata 12 Base Reference Manual, College Station, TX, Estados Unidos).

\section{Consideraciones éticas}

Este proyecto fue aprobado por el comité de Ética de Investigación en Humanos de la Fundación Clínica Valle de Lili, según acta No. 277-2017 del 3 de mayo de 2017. Todos los investigadores manifestaron no tener conflicto de intereses.

\section{RESULTADOS}

En el estudio se evaluaron 232 historias clínicas de pacientes con VHC tomado por diagnóstico CIE-10, quienes consultaron al servicio de consulta externa de gastrohepatología de la Fundación Valle de Lili, entre el 1 de enero de 2011 y el 31 de diciembre de 2016. De esta población se admitieron 214 pacientes que cumplían con los criterios de inclusión y los 18 restantes se excluyeron por no poseer diagnóstico confirmado de la infección por VHC o por haber fallecido al momento de la recolección de los datos, pues la mortalidad no fue objeto del presente estudio. Las características generales de esta población se encuentran en la Tabla 1.

Tabla 1. Características generales de los pacientes con hepatitis $C$

\begin{tabular}{lc}
\hline \multicolumn{1}{c}{ Característica } & $\mathbf{n}(\%)$ \\
\hline Edad al diagnóstico (media) & $58,7 \pm 14,7$ \\
Sexo & \\
$\quad$ Femenino & $132(61,6)$ \\
$\quad$ Masculino & $82(38,32)$ \\
Pacientes con genotipificación & $58(27,1)$ \\
Cirrosis & $63(29,4)$ \\
Hepatocarcinoma & $7(3,2)$ \\
Coinfección con VHB & $4(1,8)$ \\
Coinfección con VIH & $11(5,1)$ \\
\hline
\end{tabular}

Se evaluaron también los antecedentes relacionados con el contagio del virus (Tabla 2), y se encontró que en términos generales la mayoría de los pacientes (75 \%) había sido sometido al menos a un procedimiento quirúrgico, con un promedio general de 1,34 cirugías por paciente. En relación con el antecedente de trasplante, el renal fue el más frecuente $(64,2 \%)$. El trasplante hepático se encontró con menor prevalencia y no hay claridad en las historias clínicas de si el contagio con el virus que llevó al paciente a requerir este procedimiento fue previo a la enfermedad hepática.
Tabla 2. Principales antecedentes de los pacientes con hepatitis $C$

\begin{tabular}{lc}
\hline \multicolumn{1}{c}{ Antecedentes } & $\mathbf{n}(\%)$ \\
\hline Quirúrgicos & 287 \\
Cirugías abdominales & $114(39,7)$ \\
Cirugías ginecológicas & $81(28,2)$ \\
Cirugías ortopédicas & $30(10,4)$ \\
Otras cirugías & $62(21,6)$ \\
Trasplantes & 14 \\
Trasplante hepático & $5(35,7)$ \\
Trasplante renal & $9(64,2)$ \\
Enfermedades hematológicas & 14 \\
Hemofilia & $6(42,8)$ \\
Drepanocitosis & $3(21,4)$ \\
Linfomas/leucemias & $3(21,4)$ \\
Púrpura trombocitopénica & $1(7,1)$ \\
Enfermedad de Von Willebrand & $1(7,1)$ \\
Transfusiones & $79(36,9)$ \\
Tatuajes & $13(6)$ \\
\hline
\end{tabular}

Es ampliamente reconocido que los pacientes con enfermedades hematológicas requieren trasfusión de hemoderivados de manera frecuente a lo largo de la vida. Es por ello que presentan un mayor riesgo de contagio del VHC por esta vía. En la población analizada, se encontró que el $6,5 \%$ de los pacientes presentaba alguna alteración de esta naturaleza, con la mayor prevalencia de hemofilia $(42,8 \%)$, seguida de la anemia de células falciformes $(21,4 \%)$ y de neoplasias de la línea blanca $(21,4 \%)$.

Se analizaron como factores asociados con la presencia de VHC el antecedente de tatuajes y/o transfusiones de hemoderivados. El 6,07 \% de los pacientes tenía consignado en la historia clínica algún tatuaje. Las transfusiones de hemoderivados presentaron una prevalencia de $36,92 \%$ de transfusiones entre los pacientes con infección por VHC crónica. De las 59 personas con antecedente de transfusión, la mayoría fue de sexo femenino $(64,5 \%)$. Del total de los transfundidos, el 53,16 \% se encontraba entre los 51 y los 70 años; y cerca del $25 \%$ era mayor de 70 años (Tabla 3), con lo que se concluye que los mayores de 50 años presentaron más frecuentemente un antecedente transfusional. Sin embargo, estas asociaciones no fueron estadísticamente significativas.

Tabla 3. Distribución por edad en pacientes con transfusiones y VHC

\begin{tabular}{cc}
\hline Edad (rangos) & Trasfusiones $\mathbf{n}(\%)$ \\
\hline $0-30$ & $3(3,8)$ \\
$31-50$ & $14(17,7)$ \\
$51-70$ & $42(53,2)$ \\
$>70$ & $20(25,3)$ \\
\hline
\end{tabular}

*Edad: años cumplidos al momento del diagnóstico. 
También se estimó la coinfección de VIH y VHB. Se encontraron 4 pacientes con VHB $(1,86 \%)$ y VHC de forma simultánea, y 11 pacientes con VIH (5,14\%). De estos últimos, el $100 \%$ fue de sexo masculino y solo 1 de los 4 pacientes con VHB fue de sexo femenino. La mayoría $(63,6 \%)$ de los pacientes con VIH presentaba coinfección con VHC del genotipo 1A.

La complicación más frecuentemente encontrada en los pacientes con infección crónica por VHC fue la cirrosis, con una prevalencia del $29,4 \%$ y el hepatocarcinoma se encontró en cerca del 3,3\% de los pacientes.

Con respecto a la genotipificación de los pacientes, se observó que el $75 \%$ tenía genotipo $1 \mathrm{~B}$, el $13 \%$ tenía genotipo $1 \mathrm{~A}$ y el $12 \%$ restante pertenecía a los otros genotipos (2) (Figura 1). De los 117 pacientes con genotipo 1B, la mayoría (72 individuos) nació entre 1941 y 1960. La presencia de cirrosis entre pacientes con genotipo $1 \mathrm{~B}$ fue del $67,5 \%$, con una $p$ de 0,70 .

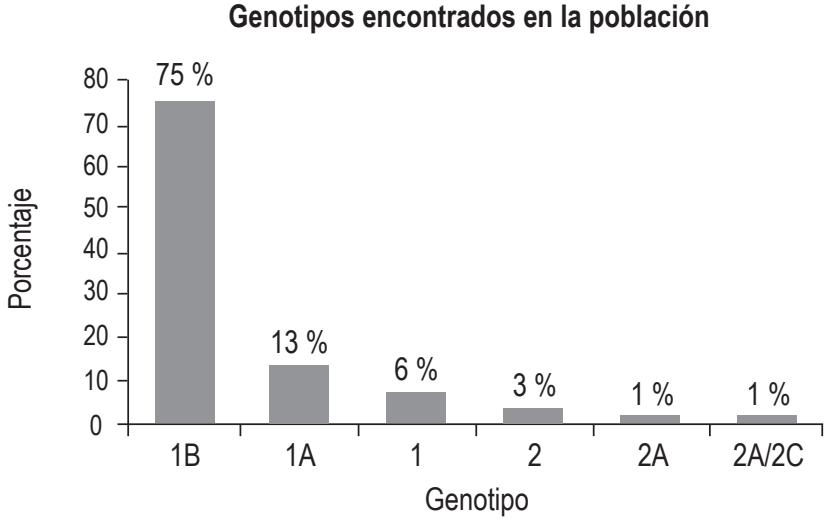

Figura 1. Se obtuvo el genotipo de 156 (73\%) pacientes.

\section{DISCUSIÓN}

En el estudio se evidenció una mayor prevalencia de mujeres infectadas $(61,6 \%)$ en nuestro medio, con una tendencia similar a la de los reportes epidemiológicos más recientes en Estados Unidos, donde el $52 \%$ de los infectados son mujeres (13). La mayoría de individuos fueron adultos en la quinta década de la vida, con la mayor proporción $(54,7$ $\%)$ de pacientes nacidos entre 1941 y 1960, a diferencia de la prevalencia encontrada en un estudio realizado por Smith y colaboradores, en el que la basta mayoría (cerca del $80 \%$ ) de los pacientes había nacido entre 1945 y 1965 (14).

Las estadísticas nacionales reportadas por el Instituto Nacional de Salud (SIVIGILA), reportaron que a nivel nacional para el 2017 se observó un predominio en la proporción del sexo masculino $(57,3 \%)$, pero estas proporciones por sexo varían según departamentos y distri- tos. Respecto a la distribución de los casos de VHC por grupo de edad, la mayor proporción reportada fue de 26,4 $\%$ para las poblaciones con edades entre 65 y más años (15). Este hallazgo es de especial importancia al evaluar los factores de riesgo para el contagio del VHC, pues antes de los noventa en Colombia no se realizaba tamizaje de rutina para VHC de los hemoderivados en los bancos de sangre; como lo reportaron de la Hoz y colaboradores en el consenso colombiano de VHC (16), la probabilidad de contraer el virus entre las personas transfundidas era del 1,5 \% para 1992 en el país; por tanto, es posible que esta haya sido la fuente de contagio de una buena proporción de los pacientes nacidos en esta época.

Los tatuajes son un factor de riesgo ampliamente reconocido para la trasmisión del VHC, tanto por el uso de las agujas contaminadas, como por las lesiones en piel y mucosas, que dejan expuestas las barreras epiteliales a la infección por el virus. En nuestra población se evidenció que cerca del $6 \%$ de los individuos tenía entre sus antecedentes los tatuajes. Sin embargo, en las historias clínicas no está consignado con claridad el lugar en el que se realizó, el momento (si fue antes o después del diagnóstico de la infección), ni las condiciones de bioseguridad bajo las cuales se realizó. Esta información es de gran valor epidemiológico, pero fue difícil precisarla pues muchos pacientes fueron atendidos previamente en otras instituciones, donde esta información no se consigna de forma rutinaria. En las estadísticas nacionales (SIVIGILA 2017), se encontró una mención general de este importante factor de riesgo y en la ficha epidemiológica de reporte obligatorio nacional (código INS 340) se indaga sobre procedimientos estéticos, piercings y tatuajes, pero no hay un lugar para el registro de las circunstancias sobre las cuales se realizaron dichos procedimientos; por ende, es difícil reportar con mayor detalle datos que relacionen de forma estrecha la infección con el tipo de tatuaje y las condiciones bajo las cuales se efectuó, siendo esta una limitante del estudio.

Un factor asociado con la infección crónica importante en nuestra institución fue la trasfusión de hemoderivados, con una prevalencia del $36,9 \%$. Esto coincide con lo encontrado en otras regiones del país, como lo descrito por Yepes y colaboradores en un estudio de casos y controles realizado en la costa caribe colombiana, donde se evidenció un Odds ratio (OR) significativo para este factor de riesgo (17). En el presente estudio se observó, además, una proporción de mujeres transfundidas ligeramente más alta (64,5\%), que en su mayoría tenía más de 50 años. Sin embargo, al evaluar cuidadosamente los datos, la mayoría de las mujeres en esta población fue sometida a cirugías con bajo riesgo de sangrado, siendo más frecuente la histerectomía en el 55,5\% de las pacientes. Este procedimiento 
presenta tasas bajas de hemorragia perioperatoria que van entre el $1 \%$ y $2 \%$, y pueden ser menores dependiendo del abordaje quirúrgico utilizado (18).

En la población estudiada, el factor de riesgo más importante encontrado fue el antecedente quirúrgico, observándose una prevalencia del $75 \%$ entre los pacientes con VHC. La mayoría de estos se había sometido a más de un procedimiento quirúrgico, con un total de 287 cirugías de diversa índole consignadas en las historias clínicas. Cabe resaltar que muchos de ellos no tenían consignados otros antecedentes o factores de riesgo para el contagio en su primera consulta a hepatología por la infección, por lo que se considera que esta podría ser una fuente significativa de transmisión del virus. Frente a este planteamiento es importante aclarar que es difícil establecer una relación causal, pues el diseño descriptivo del presente estudio no lo permite, su naturaleza retrospectiva hace difícil determinar si el momento de la cirugía fue realmente previo al contagio viral y si hay otros factores como el tiempo quirúrgico, el sangrado y el tipo de cirugía que favorecen la infección.

Otro punto importante es la incidencia de enfermedades hematológicas con alto requerimiento de trasfusiones entre los pacientes infectados. En nuestra institución se encontró este antecedente en el 6,5\% de la población, siendo la hemofilia la patología más frecuente, posiblemente por su alto riesgo de sangrados mayores.

Con respecto al genotipo, hay estudios colombianos previos que soportan los hallazgos del presente estudio, en el que el genotipo más frecuente fue el $1 \mathrm{~B}$ en cerca del $75 \%$ de los pacientes, y el segundo fue el $1 \mathrm{~A}$ en el $12 \%$. Santos, $y$ colaboradores reportaron en un estudio publicado en el 2017 un $70 \%$ de pacientes con genotipo 1 B y un $13,5 \%$ con genotipo 1A; en ese estudio, el objetivo fue describir los genotipos y subtipos del VHC en pacientes colombianos infectados (19). Adicionalmente, describieron que este hallazgo es importante debido a que el genotipo $1 \mathrm{~B}$ se ha relacionado estrechamente con la aplicación de hemoderivados y procedimientos invasivos en los que ocurren sangrado y manipulación de mucosas. Lo anterior posiblemente estaba asociado con una inadecuada implementación de las medidas de bioseguridad, por lo que se concluye este podría ser el principal factor asociado con la transmisión de la enfermedad en Colombia. Otra observación importante con respecto al genotipo es el hecho de que los nuevos tratamientos antivirales directos han demostrado una tasa de éxito mayor contra el subtipo $1 \mathrm{~B}$, por lo que más del $70 \%$ de los infectados en Colombia podría recibir un tratamiento efectivo y libre de interferón.

La coinfección con HIV y el VHC cobra especial importancia al momento de realizar un adecuado abordaje diagnóstico y terapéutico en estos pacientes. En un metaanálisis de cerca de 780 estudios evaluando las poblaciones infectadas con VIH alrededor del mundo, la prevalencia general de la coinfección con VHC se estimó en cerca del $6 \%$ (20). En nuestro medio se encontró una prevalecía del $5,14 \%$ con VIH, muy cercana a los datos epidemiológicos encontrados en la población mundial. En Colombia se ha documentado una alta prevalencia de la coinfección, especialmente en pacientes usuarios de drogas endovenosas; principalmente en Armenia, Cúcuta y Bogotá, ciudades en las que se realizaron estudios en pacientes usuarios de estos psicotóxicos $(21,22)$. En estos 2 estudios se encontraron como principales factores de riesgo las relaciones sexuales entre hombres del mismo sexo, la falta de uso de preservativo y la promiscuidad. A pesar de la importancia epidemiológica de estos datos, en el presente estudio no fue posible determinar el número de parejas sexuales de los individuos coinfectados, pues en las historias clínicas no se consignó esta información de forma rutinaria.

La frecuencia de usuarios de drogas endovenosas entre los pacientes que consultaron a esta institución fue de cero. Esto podría explicarse porque usualmente esta población de pacientes no consulta a nuestra institución, sino a centros de rehabilitación o de manejo específico del VIH. Es fundamental precisar que podría existir un sesgo de información, al preguntar al paciente por el antecedente de consumo de psicotóxicos de una forma general y no específicamente por drogas endovenosas. También es posible que muchos pacientes omitieran esta información a pesar de presentar el antecedente por temor a ser valorados de forma negativa. Lo anterior motiva a la Institución tanto a mejorar la calidad del registro de los factores de riesgo en las historias clínicas como a establecer una serie de preguntas específicas (momento y duración de la exposición, número de parejas sexuales, entre otras) en aquellos pacientes identificados como poblaciones vulnerables.

En la población a estudio se encontró un 1,86 \% de pacientes coinfectados con VHB. Esta prevalencia podría explicarse por el hecho de que muchos pacientes coinfectados carecen de marcadores serológicos para la infección por VHB. Lo anterior se ilustró en un estudio en el cual el $33 \%$ de 200 pacientes infectados con VHC tenía antígeno de superficie para VHB (HBsAg) negativo y carga viral detectable para VHB. La razón por la cual algunos pacientes con VHC que están infectados con VHB carecen de HBsAg es desconocida. Sin embargo, los datos encontrados en este estudio no discrepan mucho de lo referido en informes de prevalencia de la coinfección por otros autores (11). Se plantea que en este grupo de pacientes con infección por el VHC sería pertinente estudiar tanto la presencia del antígeno de superficie para VHB como la carga viral.

Las prevalencias de cirrosis y hepatocarcinoma encontradas en la población de estudio fueron muy similares a lo esperado según reportes de la literatura, con un $29,4 \%$ 
de cirrosis y un 3,3\% de hepatocarcinoma. Globalmente, se espera que aproximadamente del $20 \%$ al $30 \%$ de los pacientes con VHC desarrollen cirrosis (23) y del $0 \%$ al 3 $\%$ por año, carcinoma hepatocelular $(4,5)$. En lo referente a la frecuencia de trasplantes, se observó una prevalencia del $35,7 \%$ de trasplantes hepáticos entre el grupo de los trasplantados. Lamentablemente, no fue posible establecer si todos los trasplantes se realizaron por deterioro de la función hepática secundaria a la infección por el VHC, pues en las historias clínicas esta información se consignó de manera inconsistente.

Por una parte, la principal limitación de este estudio fue la heterogeneidad en el registro de las historias clínicas consignadas por los diferentes especialistas (hepatólogos, infectólogos y gastroenterólogos); así mismo, la carencia de una notificación detallada referente a los tatuajes, trasfusiones de sangre, salud sexual, uso de psicotóxicos y antecedentes quirúrgicos; lo que lleva a replantarse la importancia del registro de esta información, ya que con esto se podrían implantar medidas de intervención a nivel de salud pública, como la inclusión de preguntas más específicas sobre estos factores de riesgo en las fichas epidemiológicas (código INS 340).

Por otra parte, la principal fortaleza fue que se trata de un estudio con un análisis epidemiológico de las variables asociadas con la infección crónica con VHC. Esta información es actual y combina tanto información serológica como las principales características clínicas de la población vallecaucana, la cual, según el último reporte del Instituto Nacional de Salud, se encuentra en el quinto lugar de incidencia de VHC en lo que va del 2018 (24) y el primer lugar en muertes atribuibles al virus en el reporte del 2017 (15). Por lo anterior, esta información sirve como base para la elaboración de estudios enfocados en el diagnóstico y tratamiento antiviral de esta entidad.

\section{CONCLUSIÓN}

Según los resultados de este estudio se puede concluir que la población colombiana evaluada presenta un comportamiento de la infección por el VHC muy similar a lo encontrado en la literatura científica. Con predomino del genotipo viral $1 \mathrm{~B}$, nuestra población de manejo es susceptible de tratamiento con los nuevos agentes antivirales directos, que son medicamentos con un mejor perfil de efectos adversos y una mayor tasa de curación. Este trabajo además permite identificar que a pesar de las políticas públicas para el manejo de hemoderivados y prácticas de bioseguridad en los procedimientos invasivos, esta ruta de infección continúa siendo el principal factor asociado con el contagio, por lo que se debe continuar trabajando en la educación y prevención de la infección. Además, se propone una búsqueda activa de aquellos pacientes con historia de trasfusiones sanguíneas antes de 1993, ojalá a nivel nacional, como la estrategia implementada en Antioquia por Arroyave y colaboradores (25), en la que se puso a prueba una estrategia de tamización para el VHC en pacientes que habían recibido transfusiones antes de 1994, y se registró una prevalencia de $6,6 \%$ para anticuerpos frente al virus y de $4,2 \%$ para VHC crónica, con lo que se demostró que es una estrategia de tamizaje efectiva. Por lo anterior se propone realizar este cribado por lo pronto de carácter institucional, y posteriormente departamental.

\section{Agradecimientos}

Los autores agradecen especialmente a la Fundación Valle de Lili y a su Centro de Investigaciones Clínicas por su apoyo y colaboración en el desarrollo de este trabajo.

\section{Conflicto de intereses}

Todos los autores declaran que no tienen conflictos de intereses.

\section{Financiación}

No se recibió ninguna financiación específica para el estudio.

\section{REFERENCIAS}

1. Cercenado Mansilla E, Cantón Moreno R. Diagnóstico microbiológico de las hepatitis víricas. Procedimientos en Microbiología Clínica. España: SEIMC; 2014.

2. Chopra S, Di Bisceglie AM, Bloom A. Clinical manifestations and natural history of chronic hepatitis $\mathrm{C}$ virus infection. UpToDate [internet] 2015 [acceso el 21 de agosto de 2017]. Disponible en: https://www.uptodate.com/ contents/clinical-manifestations-and-natural-history-ofchronic-hepatitis-c-virus-infection.

3. Thein HH, Yi Q Dore GJ, Krahn MD. Estimation of stagespecific fibrosis progression rates in chronic hepatitis $C$ virus infection: a meta-analysis and meta-regression. Hepatology. 2008;48(2):418-31. doi: 10.1002/hep.22375.

4. Hu KQ, Tong MJ. The long-term outcomes of patients with compensated hepatitis $\mathrm{C}$ virus-related cirrhosis and history of parenteral exposure in the United States. Hepatology. 1999;29(4):1311-6. doi: 10.1002/hep.510290424.

5. Fattovich G, Giustina G, Degos F, Tremolada F, Diodati G, Almasio P, et al. Morbidity and mortality in compensated cirrhosis type C: a retrospective follow-up study of 384 patients. Gastroenterology. 1997;112(2):463-72.

6. Chu CJ, Lee SD. Hepatitis B virus/hepatitis C virus coinfection: epidemiology, clinical features, viral interactions and treatment. J Gastroenterol Hepatol. 2008;23(4):512-20. doi: $10.1111 /$ j.1440-1746.2008.05384.x. 
7. Maier I, Wu GY. Hepatitis C and HIV co-infection: a review. World J Gastroenterol. 2002;8(4):577-9.

8. Mohd Hanafiah K, Groeger J, Flaxman AD, Wiersma ST. Global epidemiology of hepatitis $C$ virus infection: new estimates of age-specific antibody to HCV seroprevalence. Hepatology. 2013;57(4):1333-42. doi: 10.1002/hep.26141.

9. Wasley A, Grytdal S, Gallagher K; Centers for Disease Control and Prevention (CDC). Surveillance for acute viral hepatitis--United States, 2006. MMWR Surveill Summ. 2008;57(2):1-24.

10. Centers for Disease Control and Prevention. Viral Hepatitis Surveillance - United States, 2010. CDC [internet] 2012 [acceso el 16 de septiembre de 2018]. Disponible en: https://www.cdc. gov/hepatitis/Statistics/2010Surveillance/index.htm.

11. Cacciola I, Pollicino T, Squadrito G, Cerenzia G, Orlando $\mathrm{ME}$, Raimondo G. Occult hepatitis B virus infection in patients with chronic hepatitis C liver disease. N Engl J Med. 1999;341(1):22-6. doi: 10.1056/NEJM199907013410104.

12. Páez Rodríguez OE, Peñaloza F. I. Epidemiología. Rev Col Gastroenterol. 2012;27(Suppl 2): 4-6.

13. Suryaprasad AG, White JZ, Xu F, Eichler BA, Hamilton $\mathrm{J}$, Patel A, et al. Emerging epidemic of hepatitis $\mathrm{C}$ virus infections among young nonurban persons who inject drugs in the United States, 2006-2012. Clin Infect Dis. 2014;59(10):1411-9. doi: 10.1093/cid/ciu643.

14. Smith BD, Patel N, Beckett GA, Jewett A, Ward JW. Hepatitis $C$ virus antibody prevalence, correlates and predictors among persons born from 1945 through 1965, United States, 1999-2008 [Abstract]. Presented at The Liver Meeting; San Francisco, California. 4-8 November 2011; Abstract 241.

15. Peralta Carvajal JC. Informe de evento. Hepatitis B, C y B-D. Colombia, año 2017. Colombia: Instituto Nacional de Salud; 2017.

16. de la Hoz F. Epidemiología de la hepatitis C en Latinoamérica y Colombia. Biomedica. 2000;20(1):66-72.
17. Yepes IJ, Lince B, Caez C, De Vuono G. Risk factors for hepatitis $\mathrm{C}$ virus infection in the Colombian Caribbean coast: A case-control study. Biomedica. 2016;36(4):564571. doi: 10.7705/biomedica.v36i4.3105.

18. Parker WH, Wagner WH. Gynecologic surgery and the management of hemorrhage. Obstet Gynecol Clin North Am. 2010;37(3):427-36. doi: 10.1016/j.ogc.2010.05.003.

19. Santos O, Gómez A, Vizcaíno V, Casas MC, Ramírez MP, Olaya P. Genotipos circulantes del virus de la hepatitis C en Colombia. Biomédica. 2017;37(1):22-7. doi: 10.7705/ biomedica.v37i1.3173.

20. Platt L, Easterbrook P, Gower E, McDonald B, Sabin K, McGowan C, et al. Prevalence and burden of HCV co-infection in people living with HIV: a global systematic review and meta-analysis. Lancet Infect Dis. 2016;16(7):797-808. doi: 10.1016/S1473-3099(15)00485-5.

21. Berbesi-Fernández DY, Segura-Cardona AM, MontoyaVelez LP, Santamaría AÁ. Use of injected heroin and risk of hepatitis $\mathrm{C}$ in three cities in Colombia. Rev Bras Epidemiol. 2017;20(3):435-44. doi: 10.1590/19805497201700030007.

22. Berbesi-Fernández D, Segura-Cardona Á, Montoya-Vélez L, Castaño-Pérez GA. Hepatitis C y VIH en usuarios de drogas inyectables en Armenia-Colombia. Adicciones. 2015;27(4):246-52. doi: 10.20882/adicciones.749.

23. Alberti A, Chemello L, Benvegnù L. Natural history of hepatitis C. J Hepatol. 1999;31 Suppl 1:17-24.

24. Peralta Carvajal JC. Informe de evento. Hepatitis B, C y B-D. Colombia, período VI de 2018. Colombia: Instituto Nacional de Salud; 2018.

25. Arroyave JC, Álvarez C, Correa G, Balcázar NM, Arbeláez MP, Navas MC. Infección por el virus de la hepatitis $\mathrm{C}$ en individuos transfundidos antes de 1994 en Antioquia, Colombia. Rev Col Gastroenterol. 2014;29(4):383-9. 